\title{
Treatment of idiopathic macular hole with silicone oil tamponade
}

This article was published in the following Dove Press journal:

Clinical Ophthalmology

5 September 2012

Number of times this article has been viewed

\section{Biljana Ivanovska-Adjievska' \\ Salih Boskurt ${ }^{\prime}$ \\ Faruk Semiz' \\ Hakan Yuzer' \\ Vesna Dimovska-Jordanova ${ }^{2}$}

'European Eye Hospital, Skopje,

Macedonia, ${ }^{2}$ Clinic for Eye Diseases, University "St Cyril and Methodius",

Skopje, Macedonia
Correspondence: Biljana IvanovskaAdjievska

European Eye Hospital, ul. Sremski

Front br.I, I000 Skopje, Macedonia

Tel +38922050999

Fax +389 22034989

Email ivanovska.biljana@gmail.com
Purpose: We analyzed the anatomical and visual outcomes after surgical treatment of idiopathic macular holes with pars plana vitrectomy, internal limiting membrane (ILM) peeling using Brilliant Blue dye, and silicone oil tamponade without postoperative posturing.

Methods: This was a retrospective interventional study of 10 eyes in eight patients who underwent surgical treatment of idiopathic macular holes using pars plana vitrectomy, ILM peeling using Brilliant Blue dye, and silicone oil tamponade without postoperative posturing. The preoperative staging of macular holes and postoperative anatomic outcomes were assessed using spectral-domain optical coherence tomography.

Results: All patients were women with a mean age of $66.86 \pm 4.8$ years. In two patients, bilateral macular holes were present and both eyes were operated on. Stage 2 macular hole was diagnosed in three eyes, three eyes had stage 3, and four eyes had stage 4 macular holes. Anatomical success and closure of the macular hole was achieved in nine eyes (90\%) after one operation. In one eye, the macular hole was closed after reoperation. The preoperative mean best-corrected visual acuity (BCVA) was 0.15 decimal units (0.8 $\log$ MAR units). Until the end of the follow-up period, BCVA was 0.25 decimal units ( $0.6 \log$ MAR units). Visual acuity was improved in seven patients (70\%). In two patients (20\%), visual acuity remained at the same level, and in one eye $(10 \%)$, visual acuity decreased. Postoperatively, all patients reported a significant reduction of metamorphopsia.

Conclusion: Initial results after $20 \mathrm{G}$ pars plana vitrectomy with peeling of the ILM, use of dye (Brilliant Blue), and tamponade with silicone oil without postoperative posturing gave good anatomical and functional outcome in terms of visual acuity and reduction of metamorphopsia. Taking into account the age of the patients, this method, which does not require prolonged postoperative face-down posturing, was well tolerated by the patients. Because the anatomical and visual outcome as well as the rate of postoperative complications are comparable to those when gas is used as a tamponading agent, silicone oil tamponade can also be safely used as a first option in surgery of macular holes. However, a longer period of follow-up of the operated eyes, as well as a larger group of operated eyes, will be required to identify long-term outcomes of this surgical treatment.

Keywords: macular hole, silicone oil, ILM peeling, pars plana vitrectomy

\section{Introduction}

Full-thickness idiopathic macular holes are a relatively common cause of a significant reduction in central vision, with a prevalence of approximately 3.3 in 1000 individuals. ${ }^{1}$ Characteristically, they occur more frequently in women $(72 \%)$ in the sixth and seventh decade of life. ${ }^{2}$ Regarding the pathogenesis of idiopathic macular holes many theories have been presented, but the vitreal theory for the formation of macular 
holes was first proposed by Lister in 1912. ${ }^{3}$ In 1988, Gass proposed a classification system for idiopathic macular holes, as well as a new hypothesis for its pathogenesis which emphasizes the role of the vitreomacular tangential traction in the formation of macular holes. ${ }^{4}$ In 1995, Gas suggested an updated biomicroscopical classification and anatomical interpretation in which the formation of macular holes goes through four stages. ${ }^{5}$ Although existing theories of macular hole pathogenesis are constantly being changed and refined, nearly all confirm the predominant role of the vitreous in their formation. Since the 1990s, the development of optical coherence tomography (OCT) as a new imaging technology for visualization of retinal structures led to a revolution in diagnosis and follow-up of a large number of eye diseases and conditions. ${ }^{6-8}$ This particularly applies to the direct visualization of the vitreomacular interface, for which OCT has contributed significantly to the understanding of the traction forces that lead to structural changes in the retina, as well as insight into the etiology, pathogenesis, and differential diagnosis of several vitreomacular disorders, such as full-thickness and lamellar macular hole, ${ }^{9,10}$ macular pseudohole, ${ }^{11}$ epiretinal membrane, ${ }^{12}$ and vitreomacular traction syndrome. ${ }^{13}$

The Vitrectomy for Prevention of Macular Holes Study concluded that $96 \%$ of eyes with stage 2 macular hole are expected to progress to stage 3 and 4 , and thus the visual acuity of patients is expected to reduce by at least two lines on the Snellen visual acuity chart. ${ }^{14,15}$ Best-corrected visual acuity (BCVA) in stage 3 and 4 macular hole ranges from 0.7 to $1.3 \log$ MAR units; according to the results of randomized, prospective clinical studies, surgical treatment is recommended for macular holes in stages 2,3 , and $4 .{ }^{16-18}$

The understanding of the anatomical changes occurring on the vitreomacular interface led to the development of surgical techniques with a high rate of success. Anatomical closure of macular hole can be achieved in over $90 \%$ of cases and stabilization of the visual acuity in about $70 \%$ of surgically treated eyes. ${ }^{19,20}$ The standard surgical technique for repair of macular holes was proposed by Kelly and Wendel ${ }^{21}$ in 1991 and includes standard 3-port pars plana vitrectomy with removal of the vitreous cortex and any existing epiretinal membranes. The removal of the epiretinal membrane reduces the vitreomacular traction and allows for flattening of the edges of the macular hole and return to their anatomical position by means of an intraocular tamponade. Typically, gas is used as a tamponade agent (usually 16\% perfluoropropane, $\mathrm{C} 3 \mathrm{~F} 8$ ); therefore, after surgery, the patient is required to take a face-down position for at least 1 week (sometimes 3-4 weeks) in order to ensure contact between the gas bubble and the macular hole. Thus far, several modifications of this classical technique have been developed, which are thought to improve anatomic results and visual acuity. These include peeling of the internal limiting membrane (ILM) from the macula, ${ }^{22}$ use of different dyes for easier visualization of the ILM, ${ }^{23}$ different intraocular tamponading agents (air, gas $\mathrm{C} 3 \mathrm{~F} 8$ in several concentrations, silicone oil), and a different regiment of postoperative posturing. Many studies have been conducted for all of these modifications, with some reporting positive outcomes while others report side effects with negative influences on visual outcome. Therefore, the use of these modifications remains controversial. ${ }^{24}$

The aim of this study was to review anatomical and visual outcomes after surgical treatment of idiopathic macular holes with pars plana vitrectomy, ILM peeling using Brilliant Blue dye, and silicone oil tamponade without postoperative posturing.

\section{Materials and methods}

This retrospective study of patients examined at the European Eye Hospital in Skopje over a period of 1 year from April 2011 to April 2012 found that 30 patients (35 eyes) were diagnosed with idiopathic macular hole. In five patients, bilateral macular holes were diagnosed.

Surgical treatment of macular hole was performed in 10 eyes of eight patients. Preoperatively, for all patients, BCVA was tested, intraocular pressure was measured, and biomicroscopy of the anterior segment and indirect noncontact biomicroscopy of the fundus with $90 \mathrm{D}$ lens were conducted. The diagnosis of macular hole in all patients was confirmed using spectral-domain OCT (Topcon 3D OCT2000; Topcon, Paramus, NJ). The potential benefits and risks of surgery were explained to the patients, informed consent was obtained from all patients, and the study complied with the Declaration of Helsinki.

Inclusion criteria included stage 2, 3, or 4 idiopathic macular hole, according to Gass classification, confirmed by indirect noncontact biomicroscopy and OCT. The patients with anamnestic data of previous eye trauma, previous macular surgery, rhegmatogenous detachment of the retina with macular hole, and myopia higher than $10 \mathrm{D}$ were excluded from the study.

All surgeries were performed under local anesthesia. All patients underwent standard $20 \mathrm{G}$ three-port pars plana vitrectomy. After limbal peritomy, sclerotomies were made and $20 \mathrm{G}$ trocars were placed in the superotemporal, superonasal, and inferotemporal quadrants. After vitrectomy of the cortex, the posterior hyaloid was removed after application of 
triamcinolone acetonid. Brilliant Blue dye was then injected for better visualization of the ILM of the retina. ILM peeling was conducted using $20 \mathrm{G}$ end-gripping forceps and a scraper with dimension of at least three disc diameters over the macula. Exchange of fluid-air and air-silicone oil $1000 \mathrm{cs}$ was conducted. Sclerotomies and conjunctiva were closed with sutures. All surgeries were performed by an experienced vitreoretinal surgeon (SB). The patients were advised to not maintain any kind of forced posturing after surgery.

Patient follow-up was at 1 day, 1 week, 1 month, and 3 months postoperatively. For each control, BCVA, and intraocular pressure were measured and indirect noncontact biomicroscopy examination of the macula was conducted. Assessment of the macula was conducted using OCT at 1, 3,6 , and 9 months after surgery. After 3 months, a second operation was performed to remove the silicone oil and phaco surgery of the lens because of different stages of lens opacification. In this second surgery, after standard phacoemulsification of the lens, posterior capsulorhexis was performed; with the help of an anterior chamber maintainer, the silicone oil was completely removed from the vitreous, after which an in-the-bag intraocular lens was implanted. After the second operation, the same interval of postoperative controls was established as after the first operation. In all patients, the anatomical outcome of surgery was assessed, complications were recorded, and the functional outcome in terms of visual acuity was evaluated.

\section{Results}

Of the total number of examined patients $(12,665)$ for a period of 1 year (April 2011 to April 2012), idiopathic macular hole was diagnosed in 30 patients ( 35 eyes). The prevalence of idiopathic macular holes was 2.4 per 1000 patients. Of these patients, $20(66.7 \%)$ were female and $10(33.3 \%)$ were male. The mean age of patients was $64.67 \pm 9.7$ years. Bilateral macular holes were found in five patients $(16.66 \%)$, of which one was male and four were female. In 15 patients (1.2 per 1000 patients), vitreomacular traction and epiretinal membranes were diagnosed as predisposing factors for the formation of macular hole.

All eight patients who had surgical treatment were women aged 61 to 73 years (mean age $66.86 \pm 4.8$ years). In two patients with bilateral macular holes, surgical treatment on both eyes was performed. All 10 treated eyes were phakic. According to preoperative assessment of macular holes with OCT using Gass classification, three eyes were stage 2 macular hole, three eyes were stage 3 , and four eyes were stage 4 . In eight eyes, the duration of the macular hole was over 6 months and in 2 eyes the duration was approximately 3 months. The mean basal diameter of the macular holes was $731.4 \mu \mathrm{m}$ (232-1255 $\mu \mathrm{m})$. In nine eyes (90\%), anatomical success was achieved with closure of the macular hole (Figures 1-4). In one patient (10\%), after removal of silicone oil, reopening of the macular hole was observed and a second surgical intervention was required, after which closure of the macular hole was achieved. No postoperative posturing was required.

In all 10 eyes, subsequent combined surgery involving cataract surgery (due to different stages of lens opacification) and silicone oil removal was performed. After the combined second surgery, no postoperative complications were noted during the follow-up period. The intraocular lens remained in place with no secondary opacification because of the performed posterior capsulorhexis and no silicon oil droplets were noted because of the early and complete removal of silicone oil. Additionally, since opening of new sclerotomies and suturing were avoided during the second surgery with the removal of silicone oil through the anterior chamber, the risks from this surgery were significantly reduced and no postoperative astigmatism was noted.

BVCA preoperatively was 0.15 decimal units $(0.8$ logMAR units). By the end of the period of monitoring, BCVA was 0.25 decimal units (0.6 logMAR units). Improvement of visual acuity was registered in seven patients $(70 \%)$, visual acuity remained the same in two patients $(20 \%)$, and one patient $(10 \%)$ showed a reduction in BCVA. Of those that showed improved BCVA, two eyes were stage 2 , three were stage 3 , and four were with stage 4 macular hole. All patients preoperatively complained of the emergence of metamorphopsia and deformation of the visual image

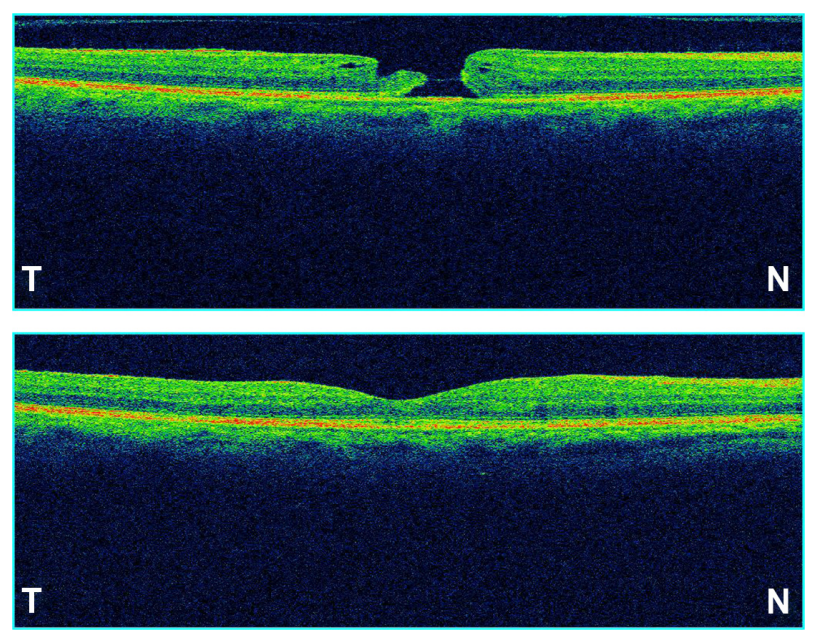

Figure I OCT of stage 3 macular hole of the right eye in a 61 -year-old patient. Preoperatively (top) and postoperatively, after removal of silicone oil (bottom). Abbreviations: OCT, optical coherence tomography; T, temporal; N, nasal. 

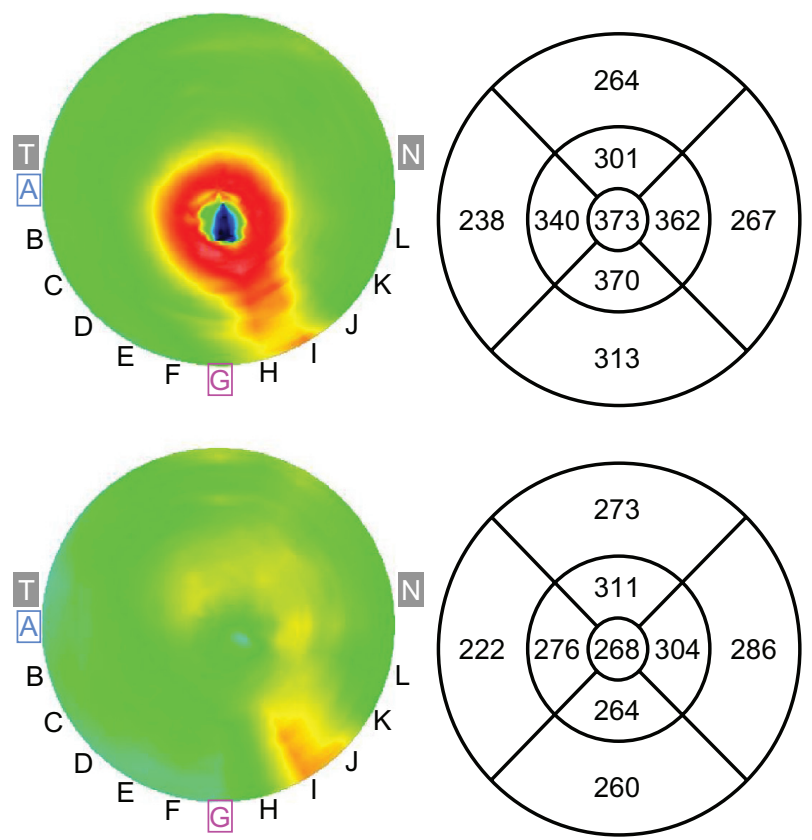

Figure 2 Retinal thickness map of the macula, with ETDRS thickness analysis in micrometers, obtained using OCT, in a patient with stage 4 macular hole. Preoperative (top) and postoperative (bottom).

Abbreviations: ETDRS, Early Treatment Diabetic Retinopathy Study; OCT, ocular coherence tomography; $\mathrm{T}$, temporal; $\mathrm{N}$, nasal.

(determined using the Amsler grid test) and inability to use their precise near vision (for reading, writing, etc); all patients were reported significant reduction of metamorphopsia and improvement of near vision postoperatively.

\section{Discussion}

The development of vitreoretinal surgery significantly improved the outcome of the treatment of macular holes in
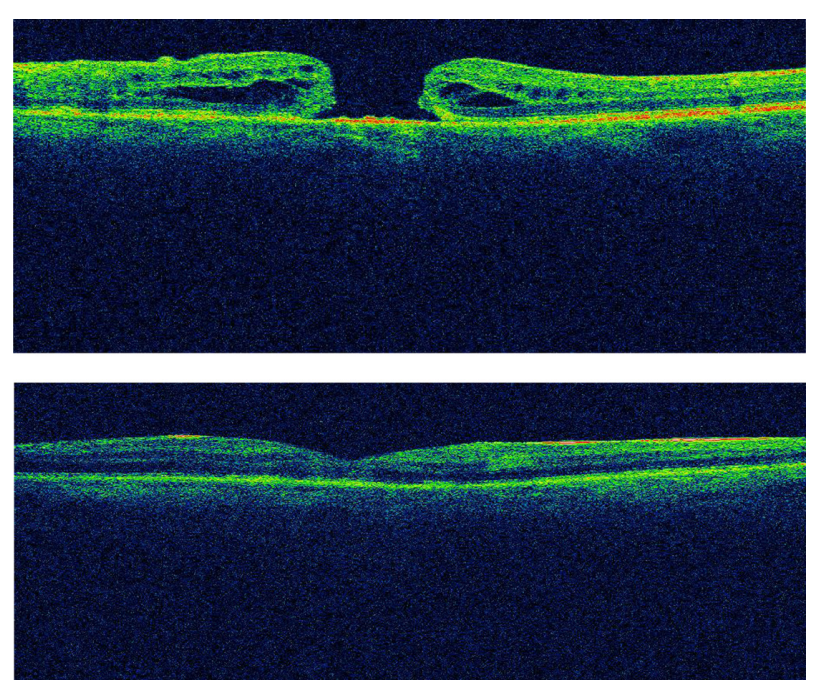

Figure 3 OCT of stage 4 macular hole of the right eye in a 70-year-old patient. Preoperatively (top) and postoperatively, after removal of silicone oil (bottom). Abbreviation: OCT, ocular coherence tomography.
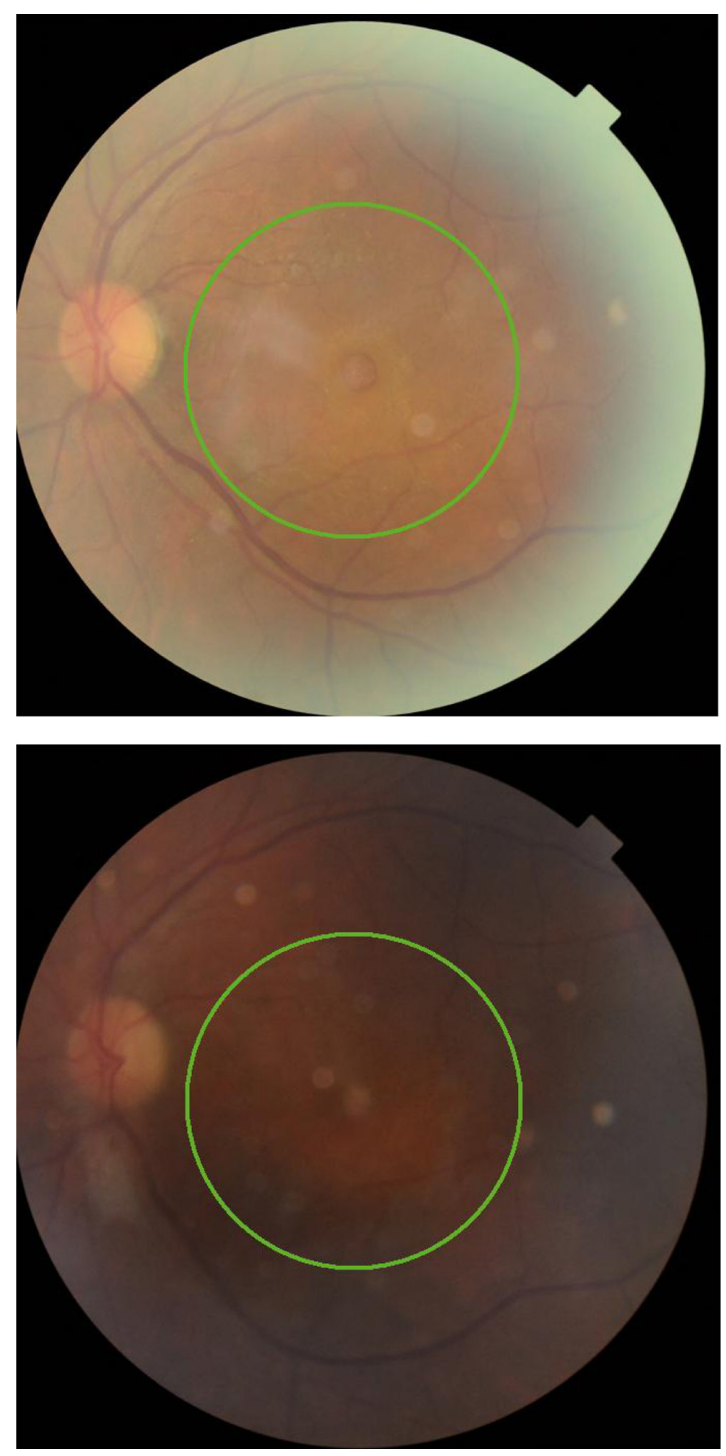

Figure 4 Fundus photography of stage 4 macular hole. Preoperatively (top) and postoperatively, after removal of silicone oil (bottom).

terms of anatomical and functional success after surgery. In previous studies, a success rate of over $90 \%$ after one surgery was presented ${ }^{25}$ as well as anatomical success of $100 \%$ in surgical treatment of macular holes in stage 2 and early stage $3 .^{26}$ According to many studies, introduction of peeling of the ILM positively affects the anatomical outcome of surgery ${ }^{27}$ primarily due to the reduction of tangential traction on the macula, although peeling of ILM is still a topic of discussion in many studies. ${ }^{28,29}$ In some studies it has been noted that peeling of ILM is associated with worse functional outcome regarding visual acuity, likely due to the damage of the inner retinal elements, ${ }^{30}$ but also because of presence of small paracentral scotoma in the visual field, likely due to minimal defects in the nerve fiber layer of the retina. ${ }^{31}$ However, most studies conclude that peeling of ILM has a beneficial effect on the anatomical 
outcome of surgery, particularly in macular holes in stages 3 and $4 .{ }^{32}$ The situation is much more clear regarding the use of dyes for better visualization of the ILM (chromovitrectomy). Particularly, dying of the ILM is extremely important for its differentiation from other structures, such as the posterior vitreous cortex and layer of retinal nerve fibers, thus avoiding possible surgical injury to this layer and resulting in poor outcome regarding visual acuity. The use of the initial choice of dye, indocyanine green, has been associated with phototoxic effects on the vitreomacular interface in many studies ${ }^{33}$ and therefore several other dyes are now in use, including Brilliant Blue, which was used in this study for dying the ILM and for which no potential toxic effects on the retina have been reported. ${ }^{34,35}$ Of particular interest in our study was the use of silicone oil as an agent for intraocular tamponade. The use of different concentrations of the gas $\mathrm{C} 3 \mathrm{~F} 8$ is standard when performing this surgery because of good anatomical and functional outcomes. However, if this gas is used, the patient must take forced posturing with the face down in long intervals after surgery (2-3 weeks), which is an unrealistic expectation for many patients due to the age of these patients (typically in the sixth or seventh decades). Recently, there has been efforts to shorten the period of postoperative posturing, ${ }^{36}$ but this sometimes results in slightly worse surgical outcomes. ${ }^{37}$ In our study, we found that the use of silicone oil as a tamponading agent is much more comfortable for the patients since no postoperative posturing is required and the patients suffer no limitations in their everyday lives (for example, traveling by plane). Our study also found that if intervention is performed by an experienced vitreoretinal surgeon with optimal filling $(90 \%)$ of the vitreous cavity, anatomical and functional results are comparable to those using gas tamponade. In some studies it was noted that the use of gas against silicone results in a better outcome of visual acuity and requires fewer interventions, ${ }^{38}$ but other studies claim the opposite. ${ }^{39,40}$ In a study using silicone tamponade but not ILM peeling, anatomical success was achieved in $80 \%$ and functional success in visual acuity improvement was achieved in only $34 \%$ of the operated eyes. In our study, anatomical success was achieved in 9 of 10 eyes $(90 \%)$; in one patient, anatomical success was achieved after the second intervention. In terms of functional outcome, improvement of visual acuity of at least $0.1 \log$ MAR units was achieved in seven eyes $(70 \%)$, in two eyes visual acuity remained the same, and in one patient, visual acuity decreased during the follow-up period. This may have been due to the improvement of surgical technique in the last 10 years, but is likely also due to the influence of peeling of the ILM in achieving good anatomical position the photoreceptors in the macula. Because the follow-up period was relatively short and varied from 3 months to 1 year, we expect further stabilization of visual function in the operated eyes.

The primary reason for choosing this type of surgery was that silicone oil tamponade compared to gas tamponade was much more comfortable for the patients, as it did not require postoperative posturing, it did not limit the everyday activities of the patients, and it achieved the same anatomical and functional results. The most frequent complication in the operations with gas is the development of cataracts, (according to some studies in which cataracts occurred in $74 \%$ of the operated eyes).$^{41}$ Hence, regardless of the type of tamponade (gas or silicone), patients operated for macular hole will require a second surgery. Therefore, the authors consider that the need for second, combined cataract/silicone-oil-removal surgery in our study did not present a greater discomfort for patients compared to the use of gas, particularly as the silicone oil was removed early (after 3 months) using a technique that does not require opening of new sclerotomies; thus, no complications were noted from the second surgery.

In our study, which is the first series examining patients with macular hole surgically treated in Macedonia, the initial results after $20 \mathrm{G}$ pars plana vitrectomy with peeling of ILM, the use of dye (Brilliant Blue), and the use of silicone oil tamponade showed satisfactory anatomical and functional outcomes in terms of visual acuity and reduction of metamorphopsia as well as improved near vision. Because of the age group of the operated patients, this method is more comfortable and acceptable to patients than the use of gas tamponade as it does not require any postoperative posturing. As the anatomical and visual outcomes, as well as the rate of postoperative complications, are comparable to those with gas tamponade, silicone oil tamponade can also be safely used as a first alternative in the surgery of macular holes, with less discomfort to the patients. However, a longer period of follow-up and a larger group of operated eyes will be required in order to assess the long-term outcomes of this surgical treatment.

\section{Disclosure}

The authors report no conflicts of interest in this work.

\section{References}

1. Fine, S. Discussion, Macular holes. Ophthalmology. 1993;100:871.

2. Evans JR, Schwartz SD, McHugh JD, et al. Systemic risk factors for idiopathic macular holes: a case-control study. Eye. 1998;12(Pt 2): 256-259.

3. Lister W. Holes in the retina and their clinical significance. $B r J$ Ophthalmol. 1924;8(1):i4-i20.

4. Gass JD. Idiopathic senile macular hole: Its early stages and pathogenesis. Arch Ophthalmol. 1988;106(5):629-639. 
5. Gass JD. Reappraisal of biomicroscopic classification of stages of development of a macular hole. Am J Ophthalmol. 1995;119(6):752-759.

6. Panozzo G, Gusson E, Parolini B, et al. Role of OCT in the diagnosis and follow up of diabetic macular edema. Semin Ophthalmol. 2003;18(2): 74-81.

7. Lalezary M, Medeiros FA, Weinreb RN, et al. Baseline optical coherence tomography predicts the development of glaucomatous change in glaucoma suspects. Am J Ophthalmol. 2006;142(4):576-582.

8. Shields CL, Materin MA, Shields JA. Review of optical coherence tomography for intraocular tumors. Curr Opin Ophthalmol. 2005;16(3): 141-154.

9. Altaweel M, Ip M. Macular hole: improved understanding of pathogenesis, staging, and management based on optical coherence tomography. Semin Ophthalmol. 2003;18(2):58-66.

10. Takahashi H, Kishi S. Tomographic features of a lamellar macular hole formation and a lamellar hole that progressed to a full-thickness macular hole. Am J Ophthalmol. 2000;130(5):677-679.

11. Haouchine B, Massin P, Tadayoni R, et al. Diagnosis of macular pseudoholes and lamellar macular holes by optical coherence tomography. Am J Ophthalmol. 2004;138(5):732-739.

12. Wilkins JR, Puliafito CA, Hee MR, et al. Characterization of epiretinal membranes using optical coherence tomography. Ophthalmology. 1996; 103(12):2142-2151

13. Gallemore RP, Jumper JM, McCuen BW 2nd, et al. Diagnosis of vitreoretinal adhesions in macular disease with optical coherence tomography. Retina. 2000;20(2):115-120.

14. de Bustros S. Vitrectomy for prevention of macular holes. Results of a randomized multicenter clinical trial. Vitrectomy for Prevention of Macular Hole Study Group. Ophthalmology. 1994;101(6):1055-1059; discussion 1060.

15. Hikichi T, Yoshida A, Akiba J, Konno S, Trempe CL. Prognosis of stage 2 macular holes. Am J Ophthalmol. 1995;119:571-575.

16. Benson WE, Cruickshanks KC, Fong DS, et al. Surgical management of macular holes. A report by the American Academy of Ophthalmology. Ophthalmology. 2001;108(7):1328-1335.

17. Kim JW, Freeman WR, Azen SP, el-Haig W, Klein DJ, Bailey IL. Prospective randomized trial of vitrectomy or observation for stage 2 macular holes. Am J Ophthalmol. 1996;121(6):605-614.

18. Freeman WR, Azen SP, Kim JW, el-Haig W, Mishell DR 3rd, Bailey I. Vitrectomy for the treatment of full-thickness stage 3 or 4 macular holes. Results of a multicentered randomized clinical trial. Arch Ophthalmol. 1997;115(1):11-21.

19. Brooks Jr HL. Macular hole surgery with and without internal limiting membrane peeling. Ophthalmology. 2000;107(10):1939-1948; discussion 1948-1949.

20. Lai MM, Williams GA. Anatomical and visual outcomes of idiopathic macular hole surgery with internal limiting membrane removal using low-concentration indocyanine green. Retina. 2007;27(4):477-482.

21. Kelly NE, Wendel RT. Vitreous surgery for idiopathic macular holes. Results of a pilot study. Arch Ophthalmol. 1991;109:654-659.

22. Tognetto D, Grandin R, Sanguinetti G, et al. Internal limiting membrane removal during macular hole surgery: results of a multicenter retrospective study. Ophthalmology. 2006;113:1401-1410.

23. Husson-Danan A, Glacet-Bernard A, Soubrane G, Coscas G. Clinical evaluation of the use of indocyanine green for peeling the internal limiting membrane in macular hole surgery. Graefes Arch Clin Exp Ophthalmol. 2006;244:291-297.

Clinical Ophthalmology

\section{Publish your work in this journal}

Clinical Ophthalmology is an international, peer-reviewed journal covering all subspecialties within ophthalmology. Key topics include: Optometry; Visual science; Pharmacology and drug therapy in eye diseases; Basic Sciences; Primary and Secondary eye care; Patient Safety and Quality of Care Improvements. This journal is indexed on Submit your manuscript here: http://www.dovepress.com/clinical-ophthalmology-journal
24. Wong D. To peel or not to peel the internal limiting membrane: a question finally answered? Br J Ophthalmol. 2009;93(8):987-988.

25. Scott IU, Moraczewski AL, Smiddy WE, Flynn HW, Feuer WJ. Long term anatomic and visual acuity outcomes after initial anatomic success with macular hole surgery. Am J Ophthalmol. 2003;135(5):633-640.

26. Margherio RR, Margherio AR, Williams GA, Chow DR, Banach MJ. Effect of perifoveal tissue dissection in the management of acute idiopathic full-thickness macular holes. Arch Ophthalmol. 2000;118(4): 495-498.

27. Lois N, Burr J, Norrie J, et al; Full-thickness Macular Hole and Internal Limiting Membrane Peeling Study (FILMS) Group. Internal limiting membrane peeling versus no peeling for idiopathic full-thickness macular hole: a pragmatic randomized controlled trial. Invest Ophthalmol Vis Sci. 2011;52(3):1586-1592.

28. Kuhn F. Point: To peel or not to peel, that is the question. Ophthalmology. 2002;109(1):9-11.

29. Hassan T, Williams GA. Counterpoint: To peel or not to peel: is that the question? Ophthalmology. 2002;109(1):11-12.

30. Smiddy WE, Feuer W, Cordahi G. Internal limiting membrane peeling in macular hole surgery. Ophthalmology. 2001;108(8):1471-1476; discussion 1477-1478.

31. Haritoglou C, Gass CA, Schaumberger M, Gandorfer A, Ulbig MW, Kampik A. Long-term follow-up after macular hole surgery with internal limiting membrane peeling. Am J Ophthalmol. 2002;134(5): 661-666.

32. Tadayoni R, Gaudric A, Haouchine B, Massin P. Relationship between macular hole size and the potential benefit of internal limiting membrane peeling. Br J Ophthalmol. 2006;90(10):1239-1241.

33. Gandorfer A, Haritoglou C, Kampik A. Retinal damage from indocyanine green in experimental macular surgery. Invest Ophthalmol Vis Sci. 2003;44(1):316-323.

34. Hisatomi T, Enaida H, Matsumoto $\mathrm{H}$, et al. Staining ability and biocompatibility of brilliant blue G: preclinical study of brilliant blue $\mathrm{G}$ as an adjunct for capsular staining. Arch Ophthalmol. 2006;124(4): 514-519.

35. Enaida H, Hisatomi T, Goto Y, et al. Preclinical investigation of internal limiting membrane peeling and staining using intravitreal brilliant blue G. Retina. 2006;26(6):623-630.

36. Almeida DR, Wong J, Belliveau M, Rayat J, Gale J. Anatomical and visual outcomes of macular hole surgery with short-duration 3-day face-down positioning. Retina. 2012;32(3):506-510.

37. Thompson JT, Smiddy WE, Glaser BM, Sjaarda RN, Flynn Jr HW. Intraocular tamponade duration and success of macular hole surgery. Retina. 1996;16(5):373-382.

38. Lai JC, Stinnett SS, McCuen BW. Comparison of silicone oil versus gas tamponade in the treatment of idiopathic full-thickness macular hole. Ophthalmology. 2003;110(6):1170-1174.

39. Karia N, Laidlaw A, West J, Ezra E, Gregor MZ. Macular hole surgery using silicone oil tamponade. Br J Ophthalmol. 2001;85(11): $1320-1323$

40. Pertile G, Claes C. Silicone oil vs gas for the treatment of full-thickness macular hole. Bull Soc Belge Ophtalmol. 1999;274:31-36.

41. Sanisoglu H, Sevim MS, Aktas B, Sevim S, Nohutcu A. Outcomes of 23-gauge pars plana vitrectomy and internal limiting membrane peeling, with brilliant blue in macular hole. Clin Opthalmol. 2011: $51177-51183$

\section{Dovepress}

PubMed Central and CAS, and is the official journal of The Society of Clinical Ophthalmology (SCO). The manuscript management system is completely online and includes a very quick and fair peer-review system, which is all easy to use. Visit http://www.dovepress.com/ testimonials.php to read real quotes from published authors. 